\title{
Dynamics of heterogeneous wetting in periodic hybrid nanopores
}

\author{
C. Picard, ${ }^{1}$ V. Gerard ${ }^{1}{ }^{2}$ L. Michel, ${ }^{1}$ X. Cattoen, ${ }^{2}$ and E. Charlaix ${ }^{1 \text { a) }}$ \\ 1) Univ. Grenoble Alpes, CNRS, LIPhy, 38000 Grenoble, France \\ ${ }^{2)}$ Univ. Grenoble Alpes, CNRS, Grenoble INP, Institut Néel, 38000 Grenoble, France \\ (Dated: 16 January 2021)
}

We present experimental and theoretical results concerning the forced filling and spontaneous drying of hydrophobic cylindrical mesopores in dynamical regime. Pores are structured with organic/inorganic moieties responsible for a periodicity of the surface energy along their axis. We find that the forced intrusion of water in these hydrophobic pores presents a slow dynamics: the intrusion pressure decreases as the logarithm of the intrusion time. We find that this slow dynamics is well described quantitatively by a classical model of activated wetting at the nanoscale, giving access to the structural length scales and surface energies of the mesoporous material.

\section{INTRODUCTION}

The wetting of micro- and mesoporous hydrophobic materials has long been studied in relation with the storage of surface energy ${ }^{1}$. The pionnering work of Eroshenko ${ }^{2,3}$, studying the intrusion and extrusion of water in hydrophobic nanopores to store and retrieve mechanical energy, started a very active field of research on the wetting and drying of lyophobic nanoporous materials, with potential applications for the design of new energy storage devices ${ }^{4-9}$.

Among the many micro- and mesoporous materials which have been studied, micelle-templated silicas (MTS's) revealed themselves as an ideal laboratory for studying wetting at a nanoscale with an ultimately simple and well-controlled geometry. The highly regular cylindrical shape of pores in materials such as MCMs or SBAs ${ }^{10,11}$ allowed to challenge in depth the concepts of macroscopic thermodynamics such as casting systems into bulk, surfaces and lines, and to confront the robustness and validity of this picture at the nanoscale? More specifically by varying the pore size of MTS's it was shown that the intrusion pressure follows the Laplace law of capillarity down to a pore radius of $1.3 \mathrm{~nm}$, validating the macroscopic concept of converting bulk energy into purely interfacial energy ${ }^{12,13}$. Similarly, the thermically activated extrusion process observed in dynamic studies, was confronted to a first-order phase transition triggered by the nucleation of a nanobubble ${ }^{12,14}$ : macroscopic capillarity provided an excellent quantitative prediction of the nucleus volume for a variety of MTS's, allowing a first experimental estimation of the sign and maximum amplitude of the water/solid/vapor line tension $^{15-18}$.

However as noted by Fraux et al in their review ${ }^{1}$, hydrophobic surfaces in real life are heterogeneous, and the question of the phase-stability and wetting behaviour of water confined between heterogeneous hydrophobic surfaces is common to a variery of materials, from biological cavities and porous polymers to inorganic and other open framework porous materials. In this article we address this question by using Periodic Mesoporous Organosilicas (PMOs) ${ }^{19}$, which are silica/organic materials combining the cylindrical pore-shape of

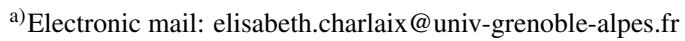

MCMs and SBAs with a periodic nano-scale alternance of the internal surface chemistry. These materials thus provide an ideally simple periodic heterogeneity. The first studies of intrusion-extrusion of water and lyophobic solutions in hydrophobized PMOS have shown their high interest for energy storage applications ${ }^{20}$. Here we specifically address the dynamics of the intrusion-extrusion processes, which is of importance to qualify the power density in energetic applications. We find that unlike MCMs and SBAs, both intrusion and extrusion pressure of water in PMOs exhibit a logarithmic kinetics. In MCMs and SBAs this logarithmic kinetics was observed only for extrusion ${ }^{15}$. We show that in PMOS, the logarithmic kinetics of both intrusion and extrusion process can be quantitatively described by a classical model of heterogeneous wetting ${ }^{21-24}$ based on thermically activated jumps of the liquid meniscus over nanoscale anchoring defects. The excellent quantitative agreement with the periodic feature of PMOS allows us to conclude that dynamic intrusion/extrusion experiments in lyophobic nanomaterials can provide a quantitative characterization of the internal heterogeneity of these nanomaterials. We discuss the relative importance of contact line pinning over nanoscale defects, and nucleation of nanobubbles/drops, for triggering the filling or drying of cylindrical mesopores.

\section{EXPERIMENTAL SECTION}

\section{A. Periodic mesoporous organosilicas as model nanopores with ordered defects}

Periodic mesoporous organosilicas (PMOs) are ordered micelle-templated matrices. Unlike micelle-templated pure silicic structure such as MCM-41, PMOs are made of several chemical-moieties organized in crystal-like pore walls which feature periodic physico-chemical properties. ${ }^{25,26}$. The PMO chosen for this study consists of independent quasi-cylindrical nanopores structured as a succession of silica/benzene annuli and first synthesized be Inagaki et al in $1999^{27}$. Pores organize as a two-dimensional hexagonal lattice (see Fig. 1). The material is synthesized following Inagaki et al (see appendix A). The nitrogen adsorption characterization leads to a specific mesopore volume of $V_{n}=0.57 \mathrm{~cm}^{3} / \mathrm{g}$ and a specific area of $760 \mathrm{~m} 2 / \mathrm{g}$ which are respectively $16 \%$ and $8 \%$ smaller than 
the one given by Inagaki potentially because of a larger number of defects in our material. From a modeling of the nitrogen adsorption isotherm ${ }^{28-30}$ we find a pore radius of $1.95 \mathrm{~nm}$ (see appendix B), while Inagaki et al indicate a radius of $1.9 \mathrm{~nm}$ for their material. From X-ray diffraction, the inter-reticular distance is $4.6 \mathrm{~nm}$ for which agrees with the value of $4.55 \mathrm{~nm}$ given by Inagaki et al. Based on this good agreement, we expect that the periodicity along the pore axis of our material presents the same periodicity of $0.77 \mathrm{~nm}$ as given by Inagaki et $\mathrm{al}^{27}$.

This native material although more hydrophobic than a purely silicic matrix requires a grafting of silica annuli to reach a hydrophobicity strong enough to be compatible with intrusion/extrusion experiments. For this purpose we follow a protocol optimized for the hydrophobization of MCM-41 based on covalent grafting of $n$-octyl-dimethylchlorosilane onto surface silanol groups (see ppendix A) ${ }^{12}$. Fitting of the same model as the one used for the native material leads to an average pore radius $R_{p}=1.5 \mathrm{~nm}$ for the grafted material (see appendix B). For a plain silica MCM-41 approximately the same size of our PMO, grafted with the very same protocol on its whole surface, Guillemot et a ${ }^{15}$ obtained a grafted pore radius of $1.34 \mathrm{~nm}$ for a pristine pore radius of $2.07 \mathrm{~nm}$. The difference between these two radii is almost twice the difference between $R_{w}$ and $R_{p}$ measured in our PMO. The reason is attributed to the fact that grafting concerns inorganic annuli only, which correspond approximately to half the total surface of the PMO, based on the simple geometric view given by Inagaki $\mathrm{et}_{\mathrm{al}} \mathrm{l}^{27}$. In other words we expect that the grafted annuli in our PMO present a radius close to the one measured in the fully grafted MCM-41 typically $R_{s}=1.3 \mathrm{~nm}$ (corresponding to regions of strong hydrophobicity) while the non grafted organic annuli (corresponding to regions of week hydrophobicity) would keep the radius $R_{w}=1.95 \mathrm{~nm}$ of the native material. The radius $R_{p}$ comprised between $R_{s}$ and $R_{w}$ results from the effective portion of the surface which is silanized.

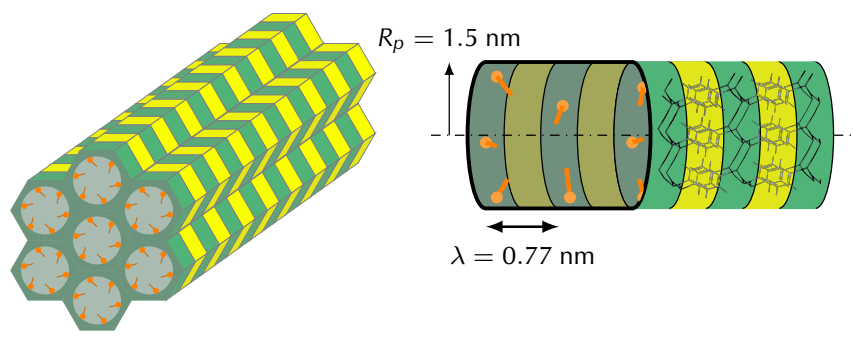

FIG. 1. Schematics of the 2D hexagonal structure of PMO with a axial periodicity of silica (green) and benzene (yellow) parts. Right : zoom on a single nanopore, the inside of which is silanized (grafting agent symbolized in orange) on silica annulus only.

The thermo-gravimetric analysis of the material, leads to an average grafting agent density on the wall of $0.7 \mathrm{~nm}^{-2}$ based on the total native material surface. Considering that silica annuli stand for half the surface of the pore this leads to a grafting density of $1.4 \mathrm{~nm}^{-2}$. This value is close to the value of $1.3 \mathrm{~nm}^{-2}$ given by Lefevre et $a l^{13}$ obtained when grafting pure silicic MCM-41. This suggest that approximately half

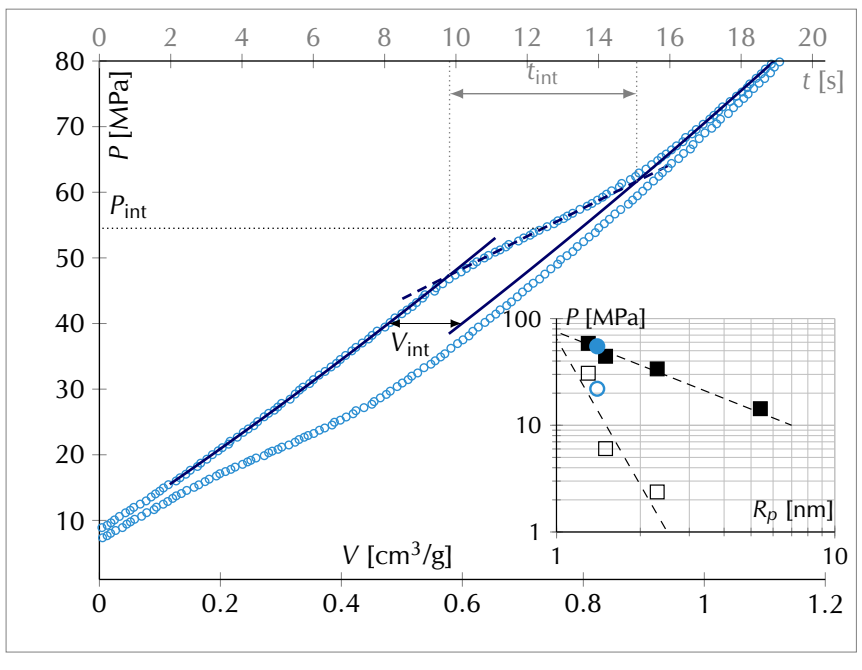

FIG. 2. Raw intrusion/extrusion isotherm of water in silanized PMO for $1 \mathrm{~g}$ of porous material. Markers are measured pressure according to volume reduction (bottom axis) or time for the compression stage (top axis). Full lines are fitting of compression before and after intrusion. The dashed line is a linear fitting of the intrusion plateau. Inset : comparison of the intrusion (full symbols) and extrusion (open symbols) pressures for water/MCM-41 (black symbols) from Lefevre et $\mathrm{al}^{12}$ and for water/PMO (blue symbols) used in this work. Dahsed lines are guides for the eye.

of the PMO surface is indeed grafted in agreement with the expected structure of the pores.

\section{B. Water intrusion-extrusion study}

Water intrusion/extrusion experiments where performed on a home-made dynamical intrusiometer that will be fully described elsewhere. This thermally regulated apparatus, driven by a traction machine (MTS 810) is able to cover four decades of intrusion/extrusion time-scale down to $10 \mathrm{~ms}$ with an almost elastic behavior in absence of sample that is perfectly preserved on the full range of time scale ${ }^{33}$. The sample made of a suspension of $490 \mathrm{mg}$ of PMO in water is placed into a dedicated deformable container immersed in glycerol as a pressure transmitting fluid within the measurement chamber of the apparatus. A raw intrusion/extrusion cycle obtained with this sample is shown in Fig. 2. This cycle demonstrates for the first time that a silanised PMO can be used as a high pressure fully reversible Lyophobic Heterogeneous System. This result extends the behavior previously observed with another PMO type which led to irreversible behavior ${ }^{31}$. Our grafted sample, which demonstrates a good stability, has been used for more than $4 \mathrm{~h}$ of cumulative intruded time. As detailed in section $\mathrm{V}$, a slow decrease of pressures is nevertheless observed according to the time the material spends in its filled state, presumably related to the hydrolysis of silane. Three main indicators are extracted from raw intrusion/extrusion data. The first quantity is the average intrusion pressure $P_{\text {int }}$, respectively the extrusion pressure $P_{\text {ext }}$. The second quantity is the intruded volume $V$ obtained from a subtraction to the 

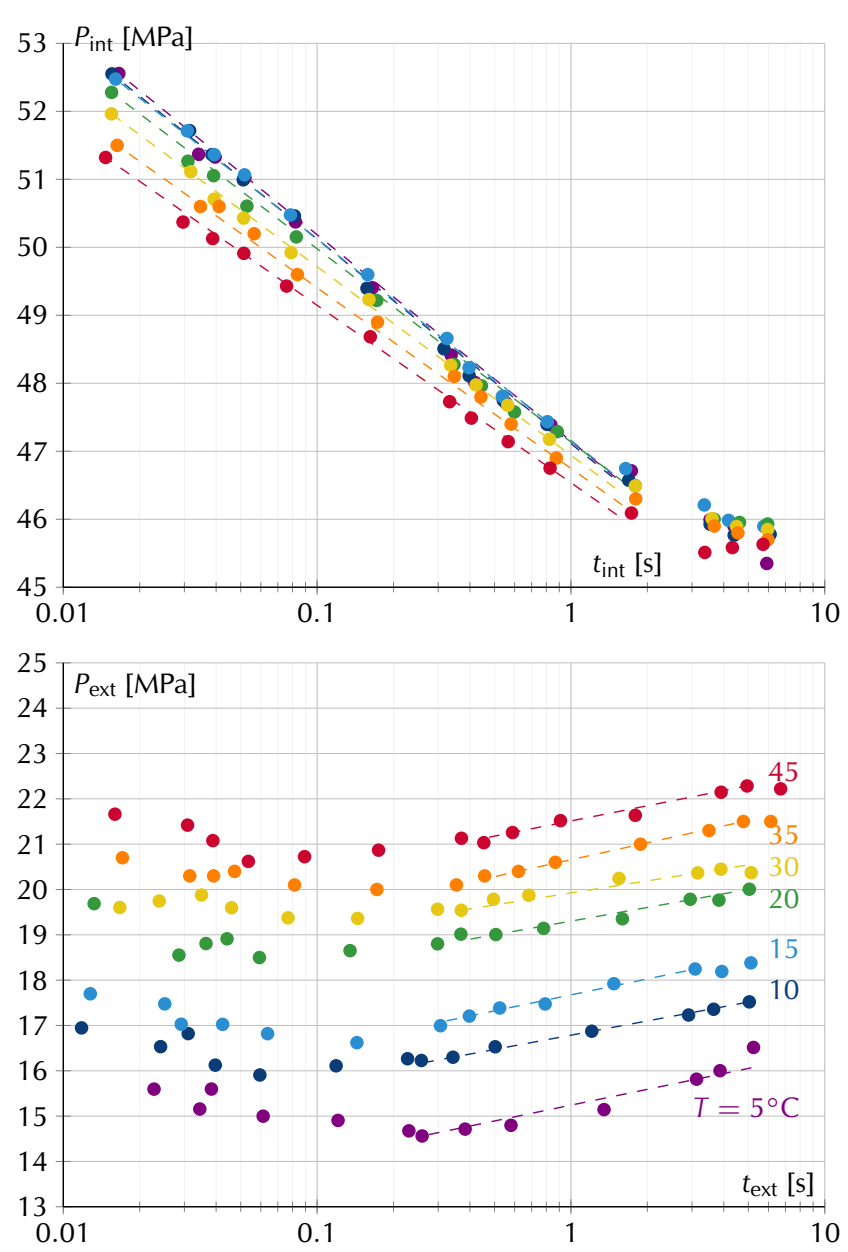

FIG. 3. Intrusion pressure $P_{\text {int }}$ (upper graph) and extrusion pressure $P_{\text {ext }}$ (lower graph) of water into PMO as a function respectively of the intrusion time $t_{\text {int }}$ and extrusion time $t_{\text {ext }}$ for temperature from $5^{\circ} \mathrm{C}$ to $45^{\circ} \mathrm{C}$ as indicated on the lower graph. Dots are experimental values, dashed line are linear fits.

raw data of the elastic deformation of the system. The third quantity is the intrusion duration $t_{\text {int }}$, respectively $t_{\mathrm{ext}}$, required to cover the intrusion/extrusion plateaus.

As shown in the inset of Fig. 2, the values of $P_{\text {int }}$ and $P_{\text {ext }}$, measured at low speed with the PMO, are in good agreement with the trend that can be interpolated from the values previously obtained with MCM- $41^{12}$.

\section{Slow dynamics of forced wetting in hydrophobized PMOS}

When exploring the dynamical regime, one observes both for intrusion and extrusion pressures a slow dependance on respective intrusion and extrusion time. The intrusion pressure follows an almost linear decrease with the logarithm of intrusion time on two decades of times, while the extrusion pressure follows an almost linear increase with the logarithm of extrusion time on approximately one decade of time (see dashed lines in Fig. 3). However, beyond this common loga- rithmic trend, several differences should be noted. First, the logarithmic trend is observed for the intrusion at short time up $2 \mathrm{~s}$ approximately while for the extrusion this behavior is valid only beyond $100 \mathrm{~ms}$. At long time for the intrusion, the decrease of $P_{\text {int }}$ seems to be slower than the logarithmic behavior at short time. At short time for the extrusion, one observe a sudden stabilization of $P_{e x t}$ to not say a non intuitive increase of $P_{e x t}$ when $t_{e x t}$ decreases. Second, the slope of the logarithmic regime is much more pronounced for the intrusion than for the extrusion. This fact contrasts with results obtained with MCM-41 with similar radius for which the opposite trend is observed, that is a stronger variation with time of $P_{\text {ext }}$ than $P_{\text {int }}$. Third, $P_{\text {ext }}$ shows a dependance of its offset on temperature which is much less pronounced for $P_{\text {int }}$. In addition one notice a slight decrease of the logarithmic slope with temperature for the intrusion, while the slope remains almost unchanged for the extrusion.

\section{THERMICALLY ACTIVATED WETTING IN HETEROGENOUS NANOPORES}

We consider the motion of a liquid meniscus in a cylinder featuring a periodic step-wise alternance of radius and wall chemistry. The pore wall is characterized by its local wettability, i.e. the local solid-vapor and solid-liquid surface tension with associated equilibrium Young's contact angle:

$$
\begin{array}{r}
\gamma_{S V, w}-\gamma_{S L, w}=\gamma_{L V} \cos \theta_{w} \\
\gamma_{S V, s}-\gamma_{S L, s}=\gamma_{L V} \cos \theta_{s} \\
\cos \theta_{s}<\cos \theta_{w} \leq 0
\end{array}
$$

The index $w$ (resp. s) refers to the pore portion which is weakly (resp. strongly) hydrophobic. The length, radius and volume of each portion are respectively $V_{s, w}=\pi R_{s, w}^{2} l_{s, w}$. The wave length of the periodic pattern $\lambda=l_{s}+l_{w}$.

The pore is connected to a liquid reservoir at pressure $P$. The free energy of the system when the liquid interface is located at a distance $x$ from the pore entrance is

$$
\begin{array}{r}
\mathscr{F}(x)=-P \int_{0}^{x} \pi R\left(x^{\prime}\right)^{2} d x^{\prime}+\int_{0}^{x} 2 \pi R\left(x^{\prime}\right) \mathscr{E}\left(x^{\prime}\right) d x^{\prime} \\
\mathscr{E}\left(x^{\prime}\right)=\left(\gamma_{S L}-\gamma_{S V}\right)\left(x^{\prime}\right)
\end{array}
$$

with $\mathscr{E}(x)$ and $R(x)$ the local surface energy and pore radius at abcissa $x$.

The equilibrium pressures $P_{i n t, o}$ and $P_{e x t, o}$ on each portion are obtained from the local condition $(d \mathscr{F} / d x)_{s, w}=0$

$$
P_{i n t, o}=-\frac{2 \gamma_{L V} \cos \theta_{s}}{R_{s}} \quad P_{\text {ext }, o}=-\frac{2 \gamma_{L V} \cos \theta_{w}}{R_{w}}
$$

The global equilibrium pressure $P_{e q}$ for which $\mathscr{F}(x)$ is periodic is defined by $l_{s}(d \mathscr{F} / d x)_{s}+l_{w}(d \mathscr{F} / d x)_{w}=0$

$$
\begin{array}{r}
P_{e q}=\frac{V_{s} P_{i n t, o}+V_{w} P_{\text {ext }, o}}{V_{s}+V_{w}}=-\frac{2 \gamma_{L V} \cos \theta_{C B}}{R_{p}} \\
R_{p}=\frac{2 V_{\text {tot }}}{A_{\text {tot }}}=\frac{V_{s}+V_{w}}{\pi R_{s} l_{s}+\pi R_{w} l_{w}}
\end{array}
$$




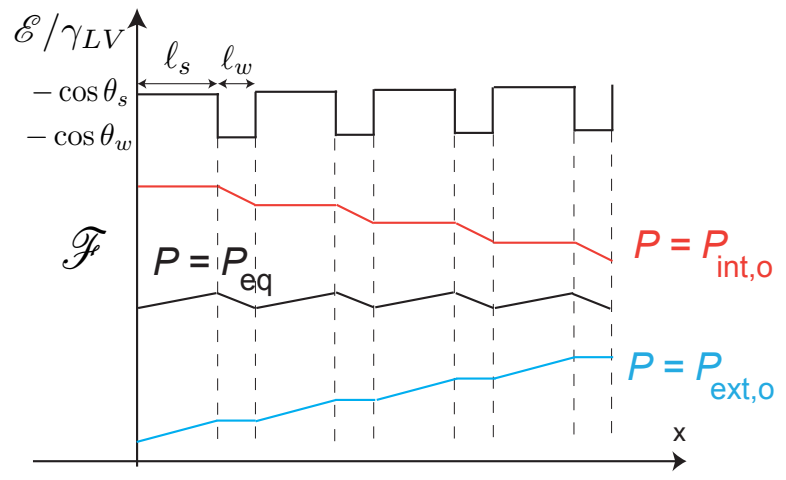

FIG. 4. Plot of eqs (4) and (5): local surface energy $\mathscr{E} / \gamma_{L V}$, and free energy $\mathscr{F}(x)$ of the system at position $x$ of the meniscus.

$R_{p}$ is the average pore size and $\theta_{C B}$ is the Cassie-Baxter contact angle.

When the reservoir pressure $P$ is equal to $P_{\text {int }, o}$ the liquid can penetrate in the pore without having to overcome any energy barrier. Symmetrically if $P=P_{\text {ext }, o}$, the liquid meniscus can freely receed up to the pore entrance. But if $P_{\text {int }, o}<P<P_{\text {ext }, o}$ the meniscus spends most of its time pinned in the local minima of the free energy, which are at the junction of a weakly hydrophobic portion on the $x^{-}$side and a strongly hydrophobic portion on the $x^{+}$side. The energy barrier $\Delta E^{+}$(resp $\Delta E^{-}$) for reaching the next minimum on the $x^{+}$side (resp $x^{-}$side) are

$$
\begin{array}{r}
\Delta E^{+}=l_{s}\left(\frac{\partial \mathscr{F}}{\partial x}\right)_{s}=V_{s}\left(P_{\text {int }, o}-P\right)>0 \\
\Delta E^{-}=-l_{w}\left(\frac{\partial \mathscr{F}}{\partial x}\right)_{w}=V_{w}\left(P-P_{\text {ext }, o}\right)>0
\end{array}
$$

It is of interest to express these barriers as a function of $P_{e q}$

$$
\begin{gathered}
\Delta E^{+}=-V_{s}\left(P-P_{e q}\right)+\mathscr{E}_{d e f} \\
\Delta E^{-}=V_{w}\left(P-P_{e q}\right)+\mathscr{E}_{d e f} \\
\mathscr{E}_{\text {def }}=V_{s}\left(P_{\text {int }, o}-P_{e q}\right)=V_{w}\left(P_{e q}-P_{e x t, o}\right)
\end{gathered}
$$

where $\mathscr{E}_{\text {def }}$ represents a defect energy. In a thermically activated process the number of jumps in the $x^{+}$direction (resp. the $x^{-}$direction) per unit time is $n^{ \pm}=v_{o} \exp \left(-\Delta E^{ \pm} / k_{B} T\right)$ where $v_{o}$ is an attempt frequency characterizing the local meniscus dynamics, and the length of each jump is $\pm \lambda$. The average liquid velocity $V=\lambda v_{o}\left(n^{+}-n^{-}\right)$writes as

$$
V=\lambda v_{o} e^{-\mathscr{E}_{d e f} / k_{B} T}\left(e^{V_{s}\left(P-P_{e q}\right) / k_{B} T}-e^{-V_{w}\left(P-P_{e q}\right) / k_{B} T}\right)
$$

A pressure value $P>P_{e q}$ corresponds to $V>0$ and a wetting process, whereas a value $P<P_{e q}$ corresponds to a drying process. It is of interest to introduce the short cut-off time $t_{o}$ and the large cross-over time $\tau$ :

$$
t_{o}=\frac{L_{P}}{\lambda v_{o}} \quad \tau=t_{o} e^{\mathscr{E}_{d e f} / k_{B} T}
$$

where $L_{P}$ is the pore length. The intrusion and extrusion times are given respectively by:

$$
\begin{aligned}
\frac{\tau}{t_{\text {int }}} & =e^{V_{s}\left(P_{\text {int }}-P_{e q}\right) / k_{B} T}-e^{-V_{w}\left(P_{\text {int }}-P_{e q}\right) / k_{B} T} \\
\frac{\tau}{t_{\text {ext }}} & =e^{V_{w}\left(P_{e q}-P_{\text {ext }}\right) / k_{B} T}-e^{-V_{s}\left(P_{e q}-P_{e x t}\right) / k_{B} T}
\end{aligned}
$$

If $P$ si significantly larger (resp. lower) than $P_{e q}$ one can neglect the exponential term of negative argument and get a purely logarithmic kinetics:

$$
\begin{array}{cl}
P_{\text {int }}=P_{\text {int }, o}-\frac{k_{B} T}{V_{s}} \ln \frac{t_{\text {int }}}{t_{o}} & P \gg P_{e q} \\
P_{\text {ext }}=P_{\text {ext }, o}+\frac{k_{B} T}{V_{w}} \ln \frac{t_{\text {ext }}}{t_{o}} \quad P \ll P_{e q}
\end{array}
$$

The slopes of the logarithmic kinetics are proportionnal to the inverse of the volume of the anchoring defects, in both directions of motion. Small size heterogeneities thus contribute to a large amplitude of the logarithmic kinetics of the intrusion and extrusion pressures.

Eq. (16) and (17) also show that the logarithmic kinetics has a limited range in time, which is the same for intrusion and extrusion. At short time $t<t_{o}$, too many activated jumps are needed to fill or empty the pores in the required time: the intrusion/extrusion processes can occur only when there is no barrier, respectively at $P_{\text {int }, o}$ and $P_{\text {ext }, o}$. Thus the cut-off time $t_{o}$ is the onset of the logarithmic kinetics. At larger times, the logarithmics kinetics saturates to the equilibrium pressure. Eq. (16) and (17) give the cross-over time for this saturation, which is equal to $\tau$, both for the intrusion and extrusion processes.

\section{COMPARISON WITH EXPERIMENTAL RESULTS: INTRUSION.}

As the logarithmic decay of the experimental intrusion pressure shows a weakening at times larger than 2s (see Fig.3), we use the full eq. (14) to compare the experimental intrusion pressure to the model of activated wetting. In principle Eq. (14) has 4 adjustable parameters: $P_{e q}, V_{s}, V_{w}$ and the cross-over time $\tau$. However we obtain only 3 reliable parameters from the fit of the intrusion pressure. The volume $V_{w}$ does not influence much the shape of the theoretical curve, and consequently it cannot be determined. More specifically we have tried 3 fitting conditions: $V_{w}$ left free to vary (but it can then take non-physical values), $V_{w}$ forced to 0 , and $V_{w}=\pi R_{P}^{2}\left(\lambda-l_{s}\right)$ assuming the value $\lambda=0.77 \mathrm{~nm}$. The values of the parameters $P_{e q}, V_{s}$ and $\tau$ found with these 3 different conditions change less than $5 \%$. Their value for the range of temperature studied is gathered in table (III) and the result of the fit is plotted in Figure (5).

\section{A. Volume of the strongly hydrophobic regions and Cassie-Baxter contact angle.}

The volume $V_{s}$ of the strongly hydrophobic portion of the pore is found to lie between $2.7 \mathrm{~nm}^{3}$ and $3.6 \mathrm{~nm}^{3}$, which cor- 


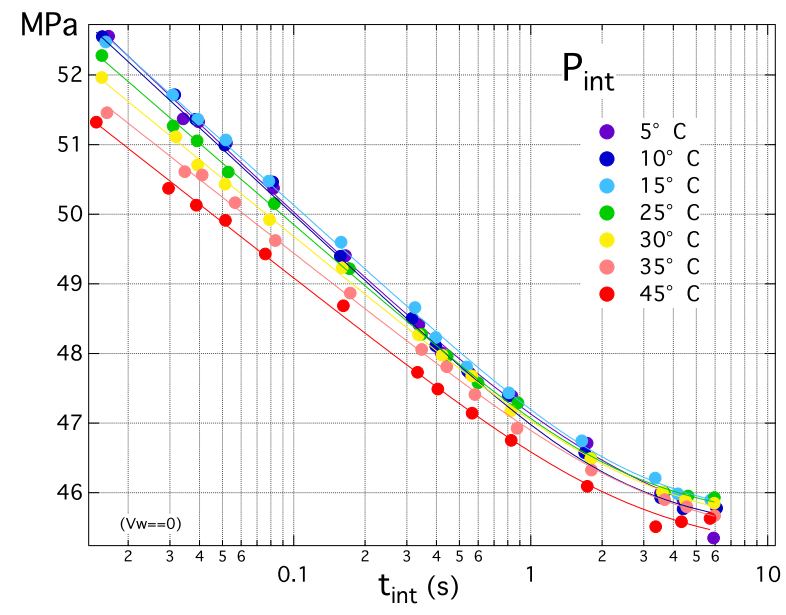

FIG. 5. Fit of the intrusion pressure for various temperatures by eq(14).

responds to a length $l_{s}=V_{s} / \pi R_{s}^{2}$ between $0.51 \mathrm{~nm}$ to $0.69 \mathrm{~nm}$. This value is compatible with the expected PMOs structure, although larger than half the period $\lambda / 2=0.385 \mathrm{~nm}$, which is the expected extension of each domains of the hybrid pores. This could be due to the finite size of the C8 silane molecules used for the grafting, which could partially extend onto the organic portion of the pores close to the organic/silica boundary. In this view, the slight increase of $l_{s}$ with the temperature, of the order of $0.16 \mathrm{~nm}$, could be associated to an increased "thickness" of the grafted-silica/organic boundary under the effect of increased thermal fluctuations.

The equilibrium pressure $P_{e q}$ gives access through Eq. (8) to the Cassie-Baxter contact angle, as well as to the average pore surface energy $\left(\gamma_{S L}-\gamma_{S V}\right)=-2 \gamma_{L V} \cos \theta_{C B}=P_{e q} R_{p} / 2$. The average pore surface energy $\left(\gamma_{S L}-\gamma_{S V}\right)$ is essentially independant of the temperature and has a value of $33.8 \pm 0.2 \mathrm{mN} / \mathrm{m}$. This quasi-absence of temperature variation is attributed to the fact that the solid interface is weakly affected by thermal fluctuations. The Cassie-Baxter contact angle is found to increase from $116.8^{\circ}$ to $119.3^{\circ}$ in the temperature range $5^{\circ} \mathrm{C}-45^{\circ} \mathrm{C}$. This variation of $\theta_{C B}$ with the temperature is a pure conse-

TABLE I. Raw parameters $V_{s}, P_{e q}$ and $\tau$ obtained from fitting the intrusion pressure with eq. (14). The length of the strongly hydrophobic parts of the pores is $l_{s}=V_{s} / \pi R_{s}^{2}$ with $R_{s}=1.3 \mathrm{~nm}$, the Cassie-Baxter contact angle $\theta_{C B}$ and the average pore surface energy $\gamma_{S L}-\gamma_{S V}=R_{p} P_{e q} / 2$ are obtained from eq. (8) with $R_{p}=1.5 \mathrm{~nm}$.

\begin{tabular}{|c|c|c|c|c|c|c|}
\hline $\begin{array}{l}\mathrm{T} \\
{ }^{\circ} \mathrm{C}\end{array}$ & $\begin{array}{c}V_{s} \\
n m^{3}\end{array}$ & $\begin{array}{c}P_{e q} \\
\mathrm{MPa}\end{array}$ & $\begin{array}{l}\tau \\
\mathrm{s}\end{array}$ & $\begin{array}{c}l_{s} \\
\mathrm{~nm}\end{array}$ & $\theta_{C B}$ & $\begin{array}{r}\gamma_{S L}-\gamma_{S V} \\
\mathrm{mN} / \mathrm{m}\end{array}$ \\
\hline 5 & 2.7 & 45.17 & 3 & 0.51 & 116.9 & 33.9 \\
\hline 10 & 2.73 & 45.1 & 3.1 & 0.52 & 117.1 & 33.8 \\
\hline 15 & 2.87 & 45.2 & 3.3 & 0.54 & 117.5 & 33.9 \\
\hline 25 & 3.08 & 45.3 & 2.83 & 0.58 & 118.1 & 34 \\
\hline 30 & 3.35 & 45.2 & 3.5 & 0.63 & 118.4 & 33.9 \\
\hline 35 & 3.56 & 45.0 & 3.8 & 0.67 & 118.7 & 33.8 \\
\hline 45 & 3.67 & 44.9 & 3.1 & 0.69 & 119.3 & 33.7 \\
\hline
\end{tabular}

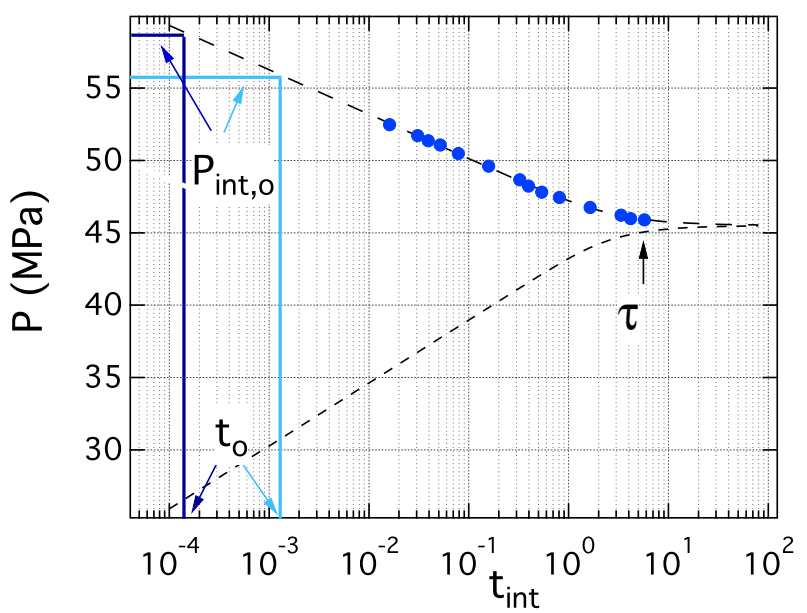

FIG. 6. Dashed line: prediction of eq. (14) for the intrusion pressure at $T=15^{\circ} \mathrm{C}$. At short time $t<t_{o}=L_{P} / \lambda v_{o}$ the intrusion pressure saturates to $P_{\text {int }, o}$. Two values of $t_{o}$ are sketched: $v_{o}=1 \mathrm{MHz}$ (light blue) giving $P_{\text {int }, o}=53.8 \mathrm{MPa}$, and $v_{o}=10 \mathrm{MHz}$ (dark blue) giving $P_{\text {int }, o}=56.8 \mathrm{MPa}$.

quence of the decrease of the water surface tension $\gamma_{L V}(T)$ with temperature, reaching $10 \%$ of relative variation in our temperature range.

Finally the properties of the weakly hydrophobic parts of the pore can be evaluated indirectly using the relation $V_{w}=$ $V_{s}\left(R_{s}^{-1}-R_{p}^{-1}\right) /\left(R_{p}^{-1}-R_{w}^{-1}\right)$ obtained from eq. (8). For the average value of the intrusion volume $V_{s}=3.2 \mathrm{~nm}$ and hydrophobic length $l_{s}=0.6 \mathrm{~nm}$, we find $V_{w}=2.13 \mathrm{~nm}, l_{w}=$ $V_{w} / \pi R_{w}^{2}=0.18 \mathrm{~nm}$, and $l_{s}+l_{w}=0.78 \mathrm{~nm}$, a value very close to the period $\lambda=0.77 \mathrm{~nm}$ first determined by Inagaki et $\mathrm{al}^{27}$.

\section{B. Time extension of the logarithmic kinetics.}

Regarding the cross-over time $\tau$, we extract from eq. (11) and (16) the following relation

$$
P_{\text {int }, o}=P_{e q}+\frac{k_{B} T}{V_{s}} \ln \frac{\tau}{t_{o}} \quad t_{o}=\frac{L_{p}}{\lambda v_{o}}
$$

The unknown parameter $t_{o}$ entering in the logarithmic term has to be estimated. We assume $\lambda=0.77 \mathrm{~nm}$, and take $L_{P}=1 \mu \mathrm{m}$ as the average length of pores in PMOs powder. The estimation of the frequency $v_{o}$ is less straightforward. Clearly a jump of the meniscus implies a displacement of the whole liquid column, and cannot be ascribed to a purely molecular mechanism. It is thus more relevant to consider the typical frequency of the liquid meniscus in the nanopore. The frequency response of a meniscus in a cylindrical capillary, studied by Gayvallet et $\mathrm{al}^{32}$, is flat up to a cut-off frequency $v_{m}=\omega_{o}^{2} / 2 \pi \omega_{c}$ where $\omega_{o}^{2}=2 \gamma_{L V} \sin \theta(1+\sin \theta)^{2} / \rho L_{P} R_{P}^{2}$ is the angular frequency of undamped oscillation, and $\omega_{c}=$ $8 \eta / \rho R_{p}^{2}$ is the angular frequency associated to the viscous damping in a cylindrical tube. Above $v_{m}$ the motion of the liquid is overdamped. Used in a PMOs nanopore with a contat angle lying between $90^{\circ}$ and $120^{\circ}$ the cut-off frequency 
TABLE II. Range of values of $P_{\text {int }, o}$ obtained for $v_{o}$ lying between 1-10 MHz. The contact angle $\theta_{s}$ on the most hydrophobic part of the pore, and the solid surface energy of this grafted part $\left(\gamma_{S L}-\gamma_{S V}\right)_{s}$, are calculated from $P_{\text {int }, o}$ using eq. (18). nm.

\begin{tabular}{lccr}
\hline \hline $\begin{array}{l}\mathrm{T} \\
{ }^{\circ} \mathrm{C}\end{array}$ & $\begin{array}{c}P_{\text {int }, o} \\
\mathrm{MPa}\end{array}$ & $\begin{array}{c}\theta_{S} \\
\mathrm{mN} / \mathrm{m}\end{array}$ & $\left(\gamma_{S L}-\gamma_{S V}\right)_{S}$ \\
\hline 5 & $56.2-59.5$ & $120 \pm 1$ & $43.4 \pm 1.2$ \\
10 & $56.3-59.6$ & $120.5 \pm 1$ & $43.5 \pm 1.2$ \\
15 & $56.2-59.4$ & $120.7 \pm 1$ & $43.3 \pm 1.2$ \\
25 & $55.6-58.7$ & $121 \pm 1$ & $42.9 \pm 1.2$ \\
30 & $55.1-58$. & $121.1 \pm 1$ & $42.4 \pm 1.2$ \\
35 & $54.7-57.4$ & $121.3 \pm 0.9$ & $42 \pm 1.2$ \\
45 & $54.3-57.1$ & $121.7 \pm 0.9$ & $41.7 \pm 1.2$ \\
\hline \hline
\end{tabular}

is $v_{m} \sim 1 \mathrm{MHz}$. At higher frequencies the liquid column is pinned by viscosity, and fluctuations of the meniscus position can occur only at the expense of local dilatation/compression of the liquid, requiring a higher potential energy.

The trial value $v_{o}=1 \mathrm{MHz}$ gives $t_{o}=1.2 \mathrm{~ms}$, compatible with our data range which shows a logarithmic kinetics upon intrusion down to the lowest investigated time of $3 \mathrm{~ms}$. Our data is not compatible with a trial frequency $v_{o}$ lower than 500 kHz. Fig. (6) plots the prediction of eq. (14) at $T=15^{\circ} \mathrm{C}$ for 2 trial values of $v_{o}, 1 \mathrm{MHz}$ and $10 \mathrm{MHz}$. These two scenarii give two values of $P_{\text {int }, o}$ which differ only of $3.3 \mathrm{MPa}$. Therefore the value of $P_{\text {int }, o}$ depends weakly on the choice made for the value of $v_{o}$. Assuming that the frequency $v_{o}$ actually lies in the range 1-10 MHz, we get a solid surface energy of the strongest hydrophobic defects $\left(\gamma_{S L}-\gamma_{S V}\right)_{s}$ in the interval 41.5 to $43.5 \mathrm{mN} / \mathrm{m}$, and a contact angle $\theta_{s}$ between $119^{\circ}$ and $123^{\circ}$. These values are slightly higher than the ones found in MCM41 of comparable pore size, with the same silanization procedure (i.e. an internal surface energy $\gamma_{S L}-\gamma_{S V}$ of $36 \mathrm{mN} / \mathrm{m}$ and a contact angle of $120^{\circ 12}$ ). We attribute this to the slighlty larger grafting density achieved in the PMOs (see section II A).

\section{Ageing of the grafted silanes}

A slow ageing of the material is observed according to the cumulated time $t_{f i l}$ the material spends at high pressure, filled in water. The upper graph in Fig. 7 shows the evolution of $P_{i n t}$ according to $t_{i n t}$ for three times $t_{f i l}$. This cumulated time $t_{f i l}$ reaches $4.3 \mathrm{~h}$ at the end of the whole set of experiments carried out with this sample. The downward shift of $P_{\text {int }}$ on the full range of considered $t_{f i l}$ is of the order of $10 \mathrm{MPa}$, that is significantly more than the modest difference in intrusion pressure measured between 5 and $45^{\circ} \mathrm{C}$. This ageing can first be characterized by a decrease of the equilibrium pressure $P_{e q}$. Based on measurement at room temperature, $P_{e q}$, initially close form $54 \mathrm{MPa}$, loses around $2 \mathrm{MPa}$ during the first cycles and then follows an almost linear decrease with a slope of $-2.3 \mathrm{MPa} / \mathrm{h}$ of cumulated filled time $t_{f i l}$ (see inset of Fig. 7 and table III). This decrease is nevertheless sufficiently slow to be able to characterize, on short time scale, the material in a quasi-identical state as illustrateded by the superimposition of two independent set of measurements for $t_{f i l}$ close from $2.5 \mathrm{~h}$. In particular, it is possible to characterize intrusion pressures for various temperatures for a quasi-identical state of the material as previously discussed. From a fit of Eq 14 on experimental dynamical pressures measured at several $t_{f i l}$ values, the decrease of $P_{e q}$ is accompanied by a decrease of $P_{\text {int }, o}$ corresponding to a decrease of $\theta_{s}$ (see table III). Considering an intermediate cut-off time $t_{o}=0.6 \mathrm{~ms}$, based on considerations detailed in section IV B, $\theta_{s}$ decrease from $125^{\circ}$ at short time to $119^{\circ}$ for $t_{f i l}=4.3 \mathrm{~h}$. This decrease, which is related to the evolution of the surface energy of the regions with stronger hydrophobicity, can be attributed to the hydrolysis of the grafting agent. Beyond the decrease of $\theta_{s}$, the fitting of experimental points reveal also a decrease of the volume $V_{s}$,
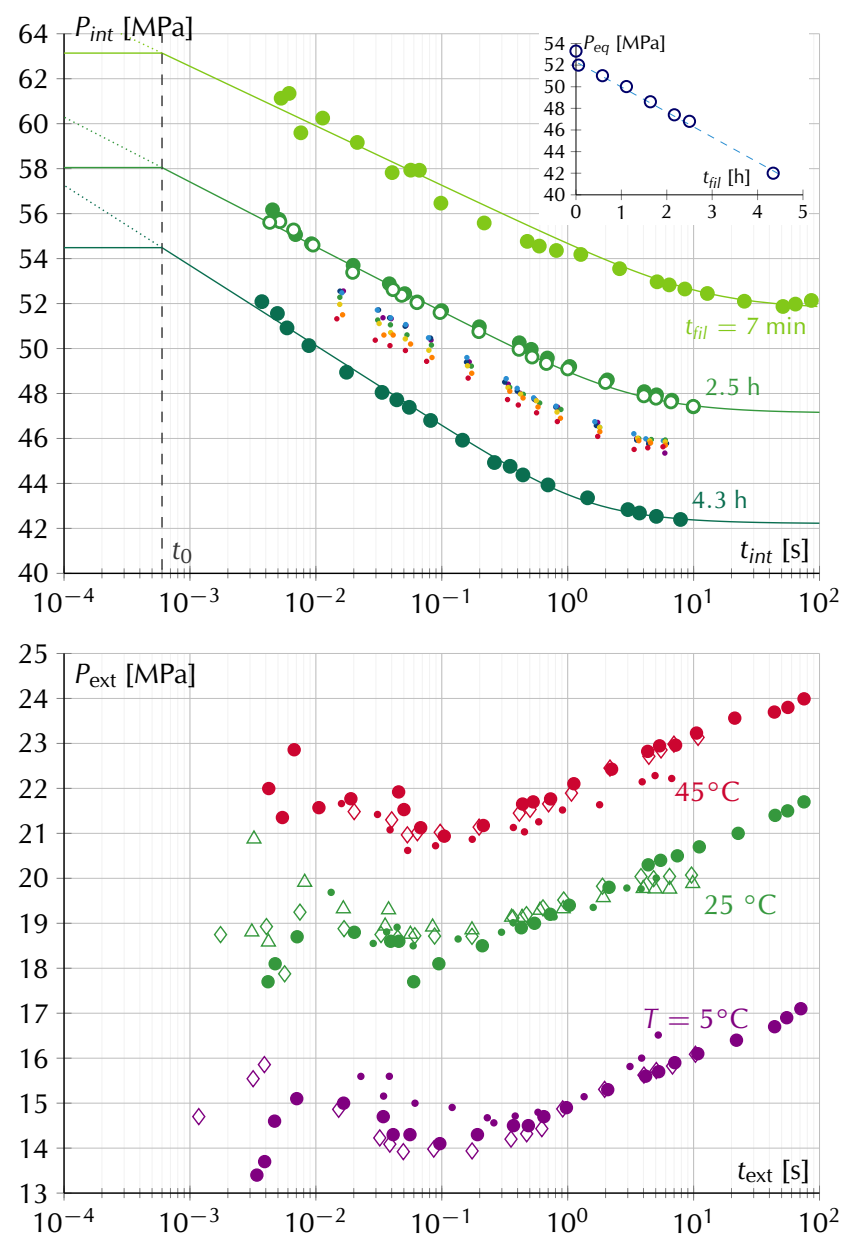

FIG. 7. Upper graph: Intrusion $P_{\text {int }}$ according to $t_{\text {int }}$ at $T=25^{\circ} \mathrm{C}$ (large green points) for three values of $t_{f i l}$ (see text). Intrusion pressures presented in Fig. 3 measured for a similar $t_{f i l}$ but various temperatures are recalled for comparison (rainbow series of small dots). Plain curves are fits of Eq. 14. Lower graph: Extrusion $P_{\text {ext }}$ according to $t_{\text {ext }}$ for $t_{f i l}<1 \mathrm{~h}$ (full circle), $t_{f i l}=2.5 \mathrm{~h}$ (open diamond) and $t_{f i l}=4.5 \mathrm{~h}$ (open triangle) and for three temperatures. Extrusion pressures presented in Fig. 3 for these three temperature are recalled for comparison (small dots). 
from $3.6 \mathrm{~nm}^{3}$ at short $t_{f i l}$ down to $2.9 \mathrm{~nm}^{3}$ for $t_{f i l}=4.3 \mathrm{~h}$. Such a decrease of $V_{s}$ corresponds to a reduction of $l_{s}$ which is also coherent with the suggested hydrolysis process.

TABLE III. Raw parameters obtained from fitting the intrusion pressure Eq (12): $V_{s}, P_{e q}$ and $\tau$. The equilibrium pressure $P_{\text {int }, o}$ and the corresponding contact angle $\theta_{s}$ are calculated using $R_{p}=1.3 \mathrm{~nm}$, and an intermediate cut-off time $t_{0}=L_{p} /\left(\lambda v_{0}\right)=0.6 \mathrm{~ms}$.

\begin{tabular}{lccccr}
\hline \hline$t_{f i l}$ & $V_{s}$ & $P_{e q}$ & $\tau$ & $P_{\text {int }, o}$ & $\theta_{s}$ \\
$\mathrm{~h}$ & $\mathrm{~nm}^{3}$ & $\mathrm{MPa}$ & $\mathrm{s}$ & $\mathrm{MPa}$ & $\circ$ \\
\hline 0.12 & 3.6 & 51.7 & 12 & 63.1 & 125 \\
2.5 & 3.3 & 47.1 & 3.67 & 58.0 & 122 \\
4.3 & 2.67 & 42.2 & 1.66 & 54.5 & 119 \\
\hline \hline
\end{tabular}

Surprisingly, unlike $P_{i n t}, P_{\text {ext }}$ is independent of $t_{f i l}$ (see lower graph in Fig 7). The extrusion pressure measured at three different temperatures for several value of $t_{f i l}$ remains the same for each temperature whatever $t_{f i l}$. This fact strongly suggest that the phenomenon which controls the dynamical extrusion pressure is different from the one which controls the intrusion pressure. Considering that $P_{i n t}$ is controlled by a thermally activated wetting, $P_{\text {int }}$ depends on the structure and physisco-chemical properties of the whole surface of the pores. A statistical degradation of the grafting is thus revealed by the measurement of $P_{i n t}$. If $P_{\text {ext }}$ is controlled by a local bubble nucleation process, as further justified in the next section, a single locus in each pore only matters. As long as some hydrophobic spots are preserved in each pores, the extrusion pressure might not be affected by the average chemical ageing of the pores. Nevertheless it is particularly striking that a slow decrease of the average hydrophobicity of the material do not impact at all the extrusion pressure (for extreme $t_{f i l}$ values, much larger than the one considered here, it is expected however that the extrusion pressure would eventually decrease when the grafting begins to be fully degrated). Another striking event that is specific to extrusion is the short time cut-off between $10^{-2} \mathrm{~s}$ and $10^{-1} \mathrm{~s}$.

\section{COMPARISON WITH EXPERIMENTAL RESULTS: EXTRUSION.}

Water extrusion from the PMOs exhibits a logarighmic kinetics at times larger than $100 \mathrm{~ms}$. The activation volumes extracted from the slope of this logarithmic characteristics (see figure 3) are listed in table IV.

Two mechanisms require in principle a thermal activation for the extrusion process to occur: the first one, already considered in homogeneous silica-tempated mesopores ${ }^{12,15}$, is the nucleation in each pore of a vapor bubble spanning the pore width, resulting in the creation of two independant meniscii. The second is the activated dewetting process studied in section (III), due to the weakly hydrophobic portions of the pore.

Regarding the qualitative differences and dissymmetries between the intrusion and extrusion logarithmic kinetics, it seems unlikely that heterogeneous dewetting is the mech-
TABLE IV. Extrusion volume $V_{\text {ext }}$, pore radius $R_{\text {extrusion }}=$ $\left(V_{\text {ext }} / 4.26\right)^{1 / 3}$, and three-phase line tension $\sigma$, derived from the extrusion pressure using eq. (20). $P_{\text {ext }, \min }$ is the minimum observed extrusion pressure.

\begin{tabular}{lcccr}
\hline \hline $\begin{array}{l}\mathrm{T} \\
{ }^{\circ} \mathrm{C}\end{array}$ & $\begin{array}{c}V_{\text {ext }} \\
\mathrm{nm}^{3}\end{array}$ & $\begin{array}{c}R_{\text {extrusion }} \\
\mathrm{nm}\end{array}$ & $\begin{array}{c}P_{\text {ext }, o} \\
\mathrm{MPa}\end{array}$ & $\begin{array}{r}\sigma \\
\mathrm{pN}\end{array}$ \\
\hline 5 & $8.37 \pm 1.5$ & $1.25 \pm 0.08$ & 19.8 & $-16 \pm 2$ \\
10 & $9.9 \pm 0.5$ & $1.32 \pm 0.03$ & 19.8 & $-18.5 \pm 2$ \\
15 & $8.12 \pm 0.4$ & $1.24 \pm 0.03$ & 20.2 & $-15 \pm 2$ \\
25 & $11 \pm 0.8$ & $1.37 \pm 0.03$ & 21.6 & $-20.5 \pm 2$ \\
30 & $13 \pm 1$ & $1.4 \pm 0.04$ & 22.5 & $-22 \pm 2$ \\
35 & $9.38 \pm 0.5$ & $1.3 \pm 0.03$ & 23.2 & $-18.5 \pm 2$ \\
45 & $10.54 \pm 0.7$ & $1.35 \pm 0.03$ & 23.5 & $-20 \pm 2$ \\
\hline \hline
\end{tabular}

anism limiting extrusion. A first dissymmetry is the temperature dependancy of the extrusion process, much larger than upon intrusion, and reminiscent to the one observed in MCM41 ${ }^{15}$. Indeed in the case of heterogeneous (de)wetting, the energy barrier involves only solid surface tensions (see eq. 11) and is thus essentially independant of the temperature. The temperature effect arises only from the Arrhenius factor and affects the slope of the logarithmic kinetics, as is indeed observed for the intrusion pressure. To the contrary the energy barrier for the bubble nucleation involves a liquid/vapor interface and is itself a temperature-dependant quantity. This was shown to contribute to a temperature effect not only on the slope of the pressure/log time characteristics, but also on its offset ${ }^{15}$.

A second dissymmetry is the short-time cut-off of the extrusion logarithmic kinetics, which appears as a strong feature disqualifying heterogeneous dewetting as a limiting mechanism. In a periodically alternating system such as the PMOs the activated wetting and dewetting process are intrinsically symmetric from the point of view of the time window, whereas no short-time cut-off is observed at intrusion.

For these reasons we compare the measured activation volume extracted from the logarithmic kinetics at extrusion, to the model of the bubble nucleation. We use here the analytic model derived by Lefevre et al $^{12}$ for a homogeneous cylindrical pore, as we are not aware of a theory taking into account heterogeneities. The energy barrier writes:

$$
\Delta \Omega=P_{\text {ext }} K_{1}(\theta) R_{p}^{3}+\gamma_{L V} K_{2}(\theta) R_{p}^{2}+K_{3}(\theta) \sigma R p
$$

where the $K_{i}$ 's are functions of $\theta$ and $\sigma$ is the 3-phase line tension, from which the extrusion pressure expresses as:

$$
P_{e x t}=\frac{k_{B} T}{K_{1}(\theta) R_{p}^{3}} \ln \frac{t_{e x t} v L}{R_{p}}-\frac{K_{2}(\theta) \gamma_{L V}}{K_{1}(\theta) R_{p}}-\frac{\sigma}{R_{p}^{2}} \frac{K_{3}(\theta)}{K_{1}(\theta)}
$$

A value $R_{\text {extrusion }}$ of the pore radius is derived from the measured activation volume $V_{\text {ext }}=K_{1}(\theta) R_{\text {extrusion. }}^{3}$. For $112^{\circ} \leq$ $\theta \leq 125^{\circ}, K_{1}(\theta)=4.26 \pm 0.02$, therefore the largest uncertainty in the evaluation of $R_{\text {extrusion }}$ comes from the experimental error bar on $V_{\text {ext }}$. The values found for $R_{\text {extrusion }}$ (see table IV) are very similar to the radius $R_{S}$ of the strongly hydrophobic part of the pore, which is coherent. This provides a further evidence of a nucleation-limited extrusion. 
As a consequence, the extrusion kinetics does not reflect the specific characteristics of the weakly hydrophobic portions of the pores. It is nevertheless possible to compare the measured values of the extrusion pressure to the indirectly evaluated nominal pressure on these weakly hydrophobic defects, according to eq (18): $P_{\text {ext }, o}=P_{e q}+k_{B} T / V_{w} \ln \left(\tau / t_{o}\right)$. With the same hypothesis than in section IV, i.e. a short cut-off time between 0.12 and $1.2 \mathrm{~ms}$, the lowest found values of $P_{\text {ext }}, o$ reported in table (IV) are slightly larger than the measured values. Therefore, once nucleated, a vapor bubble should grow and reach the entrances of the pore without need of further activation to overcome the weakest hydrophobic parts of the pores.

Finally we report the values found for the line tension $\sigma$, although its physical meaning is only indicative of an order of magnitude as the contact line crosses the chemically different portions of the pore. The negative sign and order of magnitude of 15 to $20 \mathrm{pN}$ is similar to the one found in silane-grafted templated mesoporous silicas ${ }^{15}$.

\section{CONCLUSION}

In conclusion we show that dynamic experiments of intrusion/extrusion of water in model hydrophobic nanopores provide an unprecedented approach to characterize the internal features of pore pattern, in terms of sizes and surface energies in relation with wetting dynamics at ultimate scale. In addition to a single value of the intrusion and extrusion pressure, the logarithmic variation of the pressure with the process time (or rate) allows to determine activation volumes yielding informations on the internal characteristic sizes, and further information is gained from the time window of the logarithmic kinetics.

In the silane-grafted hybrid PMOs, the logarithmic kinetics of the intrusion process is in very good agreement with a classical model of thermally activated heterogeneous wetting. We derive the size of the silane-grafted periodic portions of the PMOs, the Cassie-Baxter average wettability, as well as the contact angle of water on the grafted portions. These characteristics meet the known structural properties of PMOs, in particular the length of hydrophobic portions, identified from our mechanical characterization is compatible with the periodicity of $0.77 \mathrm{~nm}$ reported in PMO. They are also compatible with previous work on silane-grafted mesoporous micelletemplated silicas.

We also show that the extrusion process in the hybrid PMOs is governed by a vapor nucleation mechanism, and not by thermally activated heterogeneous dewetting. Due to the small size of the periodic pattern with respect to the pore radius, the nucleation process is comparable to the one studied previously in homogeneous cylindrical pores, and the critical nucleation volume is found to yield an accurate value of the pore radius.

Finally it is of interest to compare in more details the intrusion/extrusion of water in hybrid PMOs and in homogeneous MCM-41, as these model mesoporous media have a comparable nominal pore size $(1.95 \mathrm{~nm}$ for the PMOs and $2.07 \mathrm{~nm}$ for the MCM41) and are silanized using the same procedure $^{12,15}$. In Guillemot et $\mathrm{al}^{15}$ no logarithmic dynamics was observed upon intrusion, in the limit of an experimental resolution of $0.2 \mathrm{MPa} /$ decade somewhat lower than in the present work. This resolution did not allow to detect an activation lower than $50 \mathrm{~nm}^{3}$, corresponding to an average distance between potential grafting defects larger than $10 \mathrm{~nm}$. The logarithmic kinetics of intrusion evidenced in the PMOs is indeed a distinctive property of the hybrid material and its wetting heterogeneity. To the contrary the extrusion kinetics is quite similar in the two materials, from the point of view of the activation volumes and temperature dependancy. The only qualitative difference lies in the small time cut-off of the logarithmic kinetics. The latter is found here to be limited to $100 \mathrm{~ms}$ in the PMOs, whereas in the MCM-41 a limitation of the logarithmic kinetics was indeed observed at times lower than $10 \mathrm{~ms}$. We have no explanation for this difference and further work is under progress to understand the physics of the extrusion process at high rate.

\section{ACKNOWLEDGMENTS}

This work was supported by the French Research Agency (ANR LyStEn 15-CE06-0006). We are strongly grateful to Anne Galarneau who suggested initially the usage of PMOs. We thank Jérome Giraud for experimental developments and Charles Josserond for the implementation of our experiments on traction machines at SIMAP laboratory.

\section{DATA AVAILABILITY STATEMENT}

The data that support the findings of this study are available from the corresponding author upon reasonable request.

\section{Appendix A: PMOs synthesis and grafting}

Mesoporous benzene-silica PMO is synthesized following the protocol proposed by Inagaki et $a l^{27}$. A solution of $20 \mathrm{~g}$ of sodium hydroxide $(\mathrm{NaOH})$ at $6 \mathrm{M}, 8.35 \mathrm{~g}$ of octadecyltrimethylammonium (ODTMA) and $10 \mathrm{~g}$ of 1,4Bis(triethoxysilyl)benzene (BTEB) is prepared in $250 \mathrm{~mL}$ of distilled water and left for $21 \mathrm{~h}$ at room temperature under strong agitation to dissolve BTEB and homogenize its concentration. The solution, at rest, is then heated at $95^{\circ} \mathrm{C}$ for $20 \mathrm{~h}$ leading to the formation of a white precipitate $(4.1 \mathrm{~g})$ which is collected and thoroughly rinsed with pure water. Reflux extraction of the surfactant (ODTMA) is carried out at $70^{\circ} \mathrm{C}$ for $8 \mathrm{~h}$ with a solution of $250 \mathrm{~mL}$ of $95 \%$ ethanol, and $7.6 \mathrm{~mL}$ of $37 \%$ hydrochloric acid for $1 \mathrm{~g}$ of PMO powder. After surfactant extraction, the powder is dried at $40^{\circ} \mathrm{C}$ for $24 \mathrm{~h}$ and stored in vial.

To reach a high level of hydrophobicity, the silica regions of the so obtained PMO are functionnalized following Lefevre et $\mathrm{al}^{12}$. The grafting procedure starts degassing $500 \mathrm{mg}$ of $\mathrm{PMO}$ at $0.1 \mathrm{mbar}$ and $150^{\circ} \mathrm{C}$ for $20 \mathrm{~h}$. The powder is then supplemented with $20 \mathrm{~mL}$ of anhydrous toluene, $1 \mathrm{~mL}$ of 
anhydrous pyridine and $2 \mathrm{~mL}$ of octyldimethylchlorosilane (grafting agent) under argon atmosphere. The grafting last $7 \mathrm{~h}$ at $120^{\circ}$ with a slight agitation. The powder is then filtrated and thoroughly washed successively with toluene, acetone and ethanol. The powder is finally dried $2 \mathrm{~h}$ at $80^{\circ} \mathrm{C}$ and then $5 \mathrm{~h}$ at $0.1 \mathrm{mbar}$ and $100^{\circ} \mathrm{C}$ in a partially open vial.

\section{Appendix B: Pore size characterization}

The powder X-ray diffraction pattern of the so-obtained native material (see Fig. 8 is characteristic of a two-dimensional hexagonal lattice, the first peak of the spectrum correspond to an inter-reticular distance of $4.6 \mathrm{~nm}$.

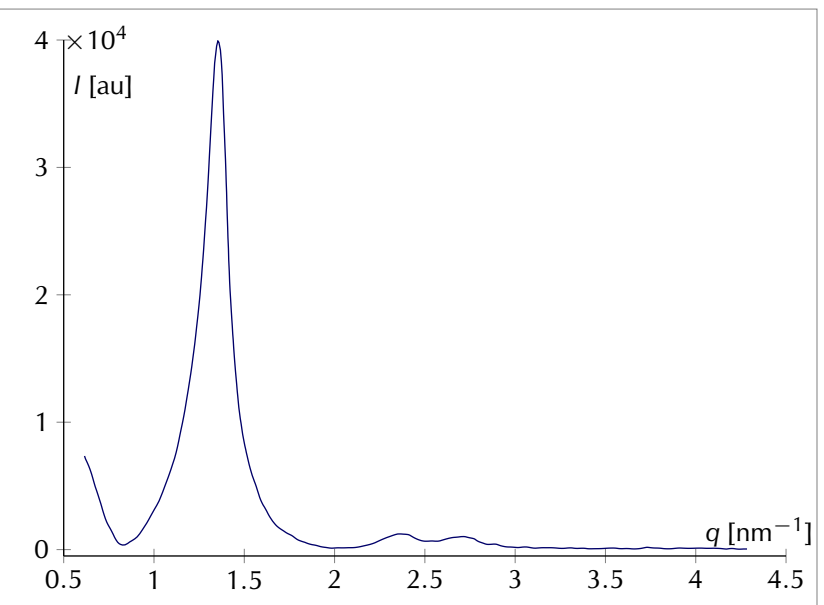

FIG. 8. X-ray diffractogram of the native PMO sample after substraction of the base line. The main pic at $q=1.35 \mathrm{~nm}^{-1}$ corresponds to an inter-reticular distance of $4.65 \mathrm{~nm}$.

The inner pore radius is deduced from nitrogen adsorption isotherms presented in Fig. 9. The model used to fit experimental points is based on the dependence of the thickness $t$ of a prewetting film according to the chemical potential imposed by the vapor pressure $p$, taking into account capillary effects and disjoining effects in cylindrical pores. Filling of a pore by capillary condensation occurs when the grand potential of the pre-wetting film becomes larger than the grand potential of the completely filled pores ${ }^{28}$. In the prewetting regime the disjoining pressure is classical modeled as $\Pi=H_{s l v} /\left(6 \pi t^{3)}\right.$ where $H_{s l v}$ is the Hamaker constant corresponding to the non retarded van der Waals interactions between the solid and the vapor through the liquid film. This model is valid only beyond a threshold thickness ${ }^{28} t_{s}=\sqrt{H_{s l v} /(4 \pi S)}$. An estimate of this thickness with $H_{s l v} \sim 10^{-19} \mathrm{~J} / \mathrm{m}^{2}$ leads to a value of $0.34 R_{p}$ in the grafted material of average radius $R_{p}$ which is close to the typical thickness measured at capillary condensation, and thus prevent the usage of the previous expression for $\Pi$ in the prewetting regime. More generally, for small mesopores, as considered here, it turns out that the disjoining pressure should rather be modeled as an exponential decay ${ }^{29,30}$. The inset of Fig. 9 shows, for the native PMO of raidus $R_{w}$, the logarithm of the dimensionless disjoining pressure $\Pi$ in the prewetting region $\left(\ln \left(p / p_{0}\right)<-1.5\right.$ with $p_{0}$ the saturation pressure) which is perfectly linear according to $t / R_{w}$. Such a behavior is precisely in agreement with a model of the form $\Pi=S / \xi \exp (-t / \xi)$ where $S$ is the spreading parameter and $\xi$ an interaction length ${ }^{29,30}$. On the contrary, trying to fit the classical $\propto t^{-3}$ model leads to the dashed line which confirms its inadequacy. Fitting of the prewetting region allows the identification of $S$ and $\xi$ and an average pore radius. We find $S=0.07 \mathrm{~J} / \mathrm{m}^{-2}, \xi=0.24 \mathrm{~nm}$ and $R_{w}=1.95 \mathrm{~nm}$ for the native material. It is worth mentioning that the so obtained $S$ and $\xi$ value are almost identical to the one obtained with a MCM41 with a radius of $2.4 \mathrm{~nm}^{30}$. For the grafted material we get $S=0.03 \mathrm{~J} / \mathrm{m}^{2}, \xi=0.25 \mathrm{~nm}$ and $R_{p}=1.5 \mathrm{~nm}$. The identified values of $R_{w}$ and $R_{p}$ imposes also the position of the capillary condensation (dashed curves in Fig. 9). This position agrees with the presumable condensation in both type of pores, nevertheless as the condensation is not sharp especially in the grafted material, the limit between the prewetting regime and the beginning of the condensation is not obvious. To be able to incorporate in the fitting procedure the points corresponding to the capillary condensation region, we consider a gaussian distribution of the pore size (which artificially helps to take also into account the presence of periodic defects). This leads to the identification of the standard deviation $\sigma$ of this distribution as an additional parameter. For the native material (plain red line in Fig. 9) we find $\sigma / R_{w}=0.15$ while for the grafted material (plain blue curve) we find a larger value $\sigma / R_{p}=0.2$ attributed to the periodic grafting.

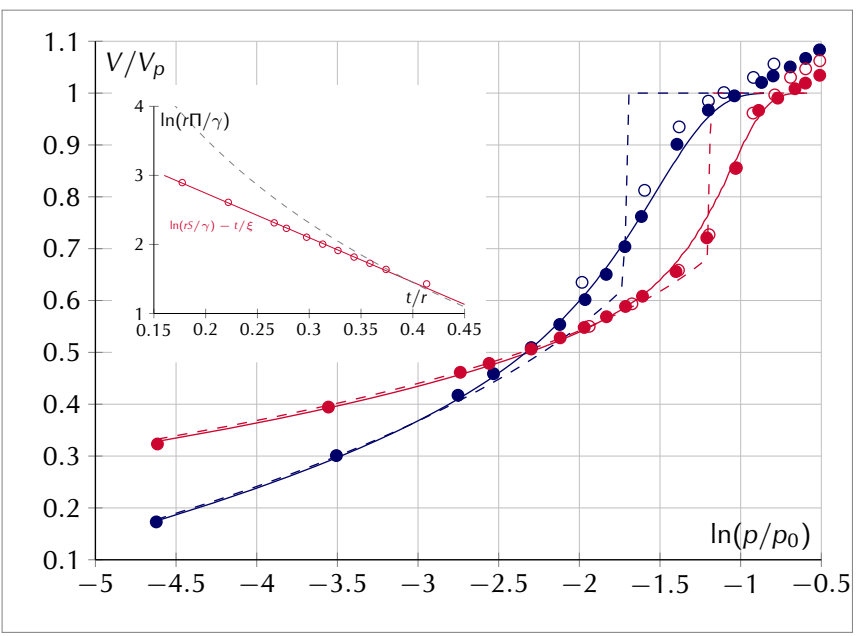

FIG. 9. Adsorption (full symbols) and desorption (open symbol) isotherms of nitrogen at $77 \mathrm{~K}$ in native material (red points) and in silanized material (blue points) with $V_{p}$ the total pore volume measured just after condensation. Lines are fits of a model of a prewetting film up to capillary condensation (see text) for a single pore size (dashed line) or gaussian pore size distribution (plain line). Inset: logarithm of the dimensionless disjoining pressure $\Pi$ according to the dimensionless film thickness in the low pressure range for the native material. Dots are experimental points. The full line is a linear fit corresponding to $\Pi=S / \xi \exp (-t / \xi)$ with $S$ the spreading parameter and $\xi$ an interaction length. Dashed line is the best fit considering $\Pi \propto t^{-3}$. 
${ }^{1}$ G. Fraux, F.-X. Coudert, A. Boutin, and A. Fuchs, Chem. Soc. Rev. 46 7421 (2017)

${ }^{2}$ V. Eroshenko and A. Fadeev, Colloid Journal 57, 446 (1995).

${ }^{3}$ A. Fadeev and V. A. Eroshenko, J. Coll. Interf. Sci. 187, 275 (1997).

${ }^{4}$ V. A. Eroshenko, R. C. Regis, M. Soulard, and J. Patarin, J. Am. Chem. Soc. 123, 8129 (2001).

${ }^{5}$ A. Han and Y. Qiao, J. Mat. Res. 22, 644 (2007).

${ }^{6}$ G. Ortiz, H. Nouali, C. Marichal, G. Chaplais, and J. Patarin, J. Phys.Chem. C 118, 7321 (2014).

${ }^{7}$ I. Khay, T. J. Daou, H. Nouali, A. Ryzhikov, S. Rigolet, , and J. Patarin, J. Phys.Chem. C 118, 3935 (2014).

${ }^{8}$ J. Canivet, A. Fateeva, Y. Guo, B. Coasne, and D. Farrusseng, Chem. Soc. rev. 43, 5594 (2014).

${ }^{9}$ M. Michelin-Jamois, C. Picard, G. Vigier, , and E. Charlaix, Phys. Rev. Lett. 115, 036101 (2015).

${ }^{10}$ J. Beck, J. Vartuli, W. Roth, M. Leonowicz, C. Kresge, K. Schmitt, C. Chu, D. Olson, E. Sheppard, S. McCullen, et al., J. Am. Chem. Soc. 114, 10834 (1992).

${ }^{11}$ D. Zhao, Q. Hui, J. Feng, B. Chmelka, and G. Stucky, J. Am. Chem. Soc. 120, 6024 (1998).

${ }^{12}$ B. Lefevre, A. Saugey, J.-L. Barrat, L. Bocquet, E. Charlaix, P. Gobin, and G. Vigier, Journal of Chemical Physics 120, 4927 (2004).

${ }^{13}$ B. Lefevre, A. Saugey, J.-L. Barrat, L. Bocquet, E. Charlaix, P. Gobin, and G. G. Vigier, Coll. and Surf. A 241, 265 (2004).

${ }^{14}$ S. Sharma and P. Debenedetti, PNAS 109, 4365 (2012).

${ }^{15}$ L. Guillemot, T. Biben, A. Galarneau, G. Vigier, and E. Charlaix, PNAS 109, 19557 (2012).

${ }^{16}$ Y. Grosu, O. Ievtushenko, V. Eroshenko, J. Nedelec, and J.-P. E. JGrolier, Coll. and Surf. A 441, 549 (2013).

${ }^{17}$ A. Tinti, A. Giacomello, and C. Casciola, Eur. Phys. J. E 41, 11658 (2018).

${ }^{18}$ M. Amabili, Y. Grosu, A. Giacomello, S. Meloni, A. Zaki, F. Bonilla, A. Faik, and C. Casciola, ACS Nano 13, 1728 (2019).

${ }^{19}$ P. Van Der Voort, D. Equivel, E. De Canck, F. Goethals, I. Van Driesshe, and F. J. Romero-Salguero, Chem. Soc. Rev. 42, 3913 (2013).

${ }^{20}$ A. Ryzhikova, T. J. Daoua, H. Noualia, J. Patarin, J. Ouwehand, S. Clerick, E. De Canck, P. Van Der Voort, and J. Martens, Microporous and Mesoporous Materials 260, 166 (2018).

${ }^{21}$ T. Blake, J. Colloid Interface Sci. 299, 1 (2006).

${ }^{22}$ E. Rolley and C. Guthman, Phys. Rev. Letter 98, 166105 (2007).

${ }^{23}$ K. Davitt, M. Pettersen, and E. Rolley, Langmuir 29, 6884 (2013).
${ }^{24}$ D. Guan, E. Charlaix, and P. Tong, Phys Rev Lett 124, 188003 (2020).

${ }^{25}$ N. Mizoshita, T. Tani, and S. Inagaki, Chemical Society reviews 40, 789 (2011), ISSN 0306-0012.

${ }^{26}$ J. G. Croissant, X. Cattoën, M. Wong Chi Man, J.-O. Durand, and N. M. Khashab, Nanoscale 7, 20318 (2015), URL http://dx.doi.org/10. 1039/C5NR05649G.

${ }^{27}$ S. Inagaki, S. Guan, T. Ohsuna, and O. Terasaki, Nature 416, 45 (2002).

${ }^{28}$ J.-M. di Meglio, D. Quere, and F. Brochard-Wyart, Compte-Rendus de l'Académie des Sciences 309, 19 (1989).

${ }^{29}$ R. J. Pellenq, B. Coasne, R. O. Denoyel, and O. Coussy, Langmuir 25, 1393 (2009), ISSN 07437463.

${ }^{30}$ I. Deroche, T. J. Daou, C. Picard, and B. Coasne, Nature Communications 10, 1 (2019), ISSN 2041-1723, URL http://dx.doi.org/10.1038/ s41467-019-12418-9.

${ }^{31}$ A. Ryzhikov, T. J. Daou, H. Nouali, J. Patarin, J. Ouwehand, S. Clerick, E. D. Canck, P. Van Der Voort, and J. A. Martens, Microporous and Mesoporous Materials 260, 166 (2018), ISSN 1387-1811, URL https: //doi.org/10.1016/j.micromeso.2017.10.037.

${ }^{32}$ H. Gayvallet and E. Charlaix, J. Phys. II France 2, 2025 (1992).

${ }^{33}$ V. Gérard, Ph.D. thesis, UGA Grenoble (2010).

${ }^{34}$ M. Bossert, A. Grosman, I. Trimaille, C. Noûs, and E. Rolley, Langmuir 36, 11054 (2020).

${ }^{35}$ E. Rolley, N. Garroun, and A. Grosman, Phys. Rev. B 95, 064106 (2017).

${ }^{36}$ S. Marchio, S. Meloni, A. Giacomello, and C. Casciola, Nanoscale 11, 21458 (2019).

${ }^{37}$ P. Giovambattista, N.and Rossky and P. Debenedetti, Phys. Rev. Lett. 102, 050603 (2009).

${ }^{38}$ G. Giovambattista, P. Debenedetti, and P. Rossky, J. Phys. Chem. C 111, 1323 (2007).

${ }^{39}$ L. Guillemot, A. Galarneau, G. Vigier, T. Abensur, and E. Charlaix, Rev. of Scientific Inst. 83, 105105 (2012).

${ }^{40}$ L. Tzanis, M. Trzpit, M. Soulard, and J. Patarin, J. Phys.Chem. C 116, 20389 (2012).

${ }^{41}$ C. T. Kresge, M. E. Leonowicz, W. J. Roth, J. C. Vartuli, and J. S. Beck, Nature 359, 710 (1992).

${ }^{42}$ P. Aryal, M. Sansom, 3, and S. Tucker, J. Mol. Biol. 427, 121 (2015).

${ }^{43}$ A. Grosman and C. Ortega, Phys. Rev. B 78, 085433 (2008).

${ }^{44}$ A. Fadeev and V. A. Eroshenko, J. Coll. Interf. Sci. 187, 275 (1997).

${ }^{45}$ A. Fadeev and V. Eroshenko, Colloid J. 58, 654 (1996). 